\title{
Effect of Silane as Coupling Agent on Dynamic Mechanical Properties of Volcanic Ash Filled PPS Composites
}

\author{
O. ÇOBAn ${ }^{a, *}$, M.Ö. BorA ${ }^{a}$, T. KutluK ${ }^{b}$, S. FidAN $^{a}$ And T. SinmazÇELIK ${ }^{c}$ \\ ${ }^{a}$ Kocaeli University, Faculty of Aeronautics and Astronautics, Kocaeli, Turkey \\ ${ }^{b}$ Kocaeli University, Chemical Eng. Dept., Kocaeli, Turkey \\ ${ }^{c}$ Kocaeli University, Mechanical Eng. Dept., Kocaeli, Turkey
}

\begin{abstract}
The purpose of this study is to investigate the effect of surface modification of volcanic ash particles on dynamic mechanical properties of volcanic ash filled polyphenylene sulfide (PPS) composites. For this purpose volcanic ash particles were modified with 1, 3, 5 vol.\% of 3-aminopropyltriethoxysilane (3-APTS) which has an organic functional group. All volcanic ash/PPS composite samples were prepared by using DSM Xplore $15 \mathrm{ml}$ twin screw microcompounder and DSM Xplore $12 \mathrm{ml}$ injection molding machines. The content of volcanic ash in composite samples was varied as 10 and $15 \mathrm{wt} \%$. Volcanic ash filler dispersion and adhesion between volcanic ash particles and PPS matrix were examined by scanning electron microscopy. Dynamic mechanical properties such as storage modulus $\left(E^{\prime}\right)$ and glass transition temperature $\left(T_{\mathrm{g}}\right)$ were investigated by TA Instruments Q800 dynamic mechanical analyzer. During the experiments, the relation between silane coupling and dynamic mechanical properties was evaluated.
\end{abstract}

DOI: 10.12693/APhysPolA.129.492

PACS/topics: 81.05.Qk

\section{Introduction}

Polyphenylene sulfide is a high-performance semicrystalline polymer that has been used in various industries such as electrical, electronic, automotive, aviation, and chemical. The reasons for its use in many products are its high mechanical properties, easy processability, excellent thermal stability, chemical resistance, flame resistance, and electro-insulating property [1-6]. However, PPS has some limitations for certain applications requiring superior flame resistance, high glass transition temperature, strength or/and toughness [7]. The addition of fillers into PPS is an attractive strategy to obtain composites with improved performance. For this reason, different inorganic particles such as $\mathrm{Al}_{2} \mathrm{O}_{3}[8], \mathrm{TiO}_{2}, \mathrm{ZnO}$, and $\mathrm{SiC}$ [9] have been melt-blended with PPS matrix. Volcanic ash (VA), which is deposited at the surface during volcanic activity, is readily accessible and has the advantage that it can be economically mined, with enormous benefits of low cost and limited negative environmental impact compared with traditional open pit quarry-type clay mining $[10,11]$. VA, known as mesoporous material, has high specific surface area, significant porosity and an appropriate pore structure, which enhances its possibility to be filled in PPS. To the best of our knowledge, very few studies deal with the effect of VA filler in polymer composites.

In our previous study possible use of VA as filler in PPS composites was investigated for its thermal and mechanical properties [10]. Because, PPS has a viscoelastic nature; its mechanical properties exhibit a

*corresponding author; e-mail: onur_coban@yahoo.com pronounced dependence on the temperature and the rate of deformation [12-14]. It was found that PPS composites had higher stiffness and energy dissipation ability with the addition of VA filler; however no remarkable change was reported for glass transition temperature. It was determined that in addition to homogeneous dispersion of fillers, strong interfacial adhesion between polymer matrix and filler is essentially crucial to obtain high-performance polymer/filler composites [15]. Surface modification of inorganic particles has attracted a great deal of attention because it produces excellent integration and improved interface between particles and polymer matrices [16-19]. For this reason determined limited enhancement in dynamic mechanical properties of PPS need to be a further investigation deal with the surface modification of VA particles. Therefore, this study aimed to investigate the effect of silane coupling of VA particles on dynamic mechanical properties of PPS composites.

\section{Materials and method}

\subsection{Materials}

PPS used as the matrix resin for the microcompounding and injection molding was Fortron 1200L1 provided by Ticona Co. The Fortron 1200L1 is an unfilled grade for extrusion applications and has a density of $1.340 \mathrm{~g} / \mathrm{cm}^{3}$. VA particles with density of $2.37 \mathrm{~g} / \mathrm{cm}^{3}$ were taken from Güneydağ tuff ring, which is located $13 \mathrm{~km}$ southwest of Nevşehir/Turkey. After scanning electron microscope (SEM) and elemental analysis the results are shown in Fig. 1 and Table I. VA samples from Güneydağ tuff ring were estimated as aphyric because of lack of minerals in the VA.

The silane coupling agent called 3-APTS is one of the most popular predilections for creating a functional group on an inorganic surface of particle. It involves a 

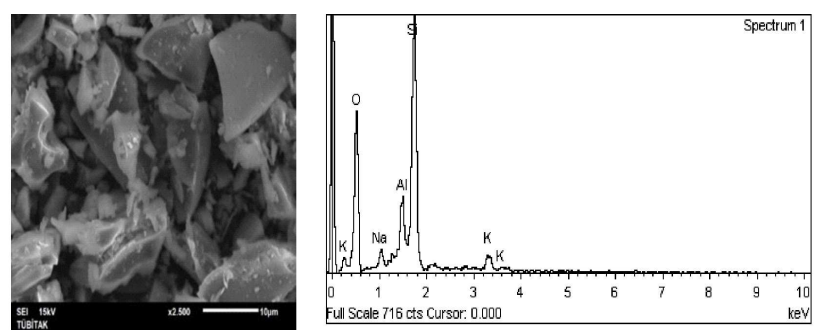

Fig. 1. SEM and elemental analysis of alkaline VA particles.

TABLE I

Elemental analysis of alkaline VA particles.

\begin{tabular}{c|c|c}
\hline \hline Elements & Weight [\%] & Atomic [\%] \\
\hline $\mathrm{O} K$ & 51.95 & 65.60 \\
$\mathrm{Na} K$ & 2.89 & 2.54 \\
$\mathrm{Al} K$ & 6.80 & 5.09 \\
$\mathrm{Si} K$ & 34.33 & 24.69 \\
$\mathrm{~K} K$ & 4.03 & 2.08 \\
Total & 100.00 &
\end{tabular}

short organic 3-aminopropyl group which cap off a primary amine. Once deposited on substrate the alkoxy groups form a covalent polymer coating with the primary amine groups bonding of the surface.

\subsection{Silane coupling}

A sum of VA whose particle size was $<71 \mu \mathrm{m}$ was added in methanol at overnight by stirring $200 \mathrm{rpm}$ to rip off from miriness and then washed with distilled water when $\mathrm{pH}$ reaches up to 7.0. Afterwards $50 \mathrm{ml}$ of $5 \mathrm{~N}$ $\mathrm{NaOH}$ solution was prepared for cleaning the VA particles. The mixture was kept in water bath more than half an hour at $100^{\circ} \mathrm{C}$. VA feculence was removed by filtration and shaked in 1 litre of distilled water for detaches the $\mathrm{NaOH}$ on the surface of VA. Pure VA feculence was dried at $110^{\circ} \mathrm{C}$ in oven at $24 \mathrm{~h}$. Powdered dry VA particles were mixed in recently prepared 3-APTS 1-3$5 \mathrm{vol} . \%(\mathrm{v} / \mathrm{v})$ in distilled water. The mixture was shaking at $75^{\circ} \mathrm{C}$ in $200 \mathrm{rpm}$ on magnetic stirrer. At last the VA feculence was filtered with whatman filter paper in filtering flask to obtain the silanized VA particles. Typical silane coupling flow sheet was shown in Fig. 2.

\subsection{Sample preparation}

All silane coated and original VA/PPS composite materials were prepared by melt compounding and injection molding processes. The content of VA in composite samples was varied as 10 and $15 \mathrm{wt} \%$. For melt compounding, DSM Xplore $15 \mathrm{ml}$ micro-compounder with a maximum processing temperature of $400^{\circ} \mathrm{C}$ and a screw speed of $250 \mathrm{rpm}$ was used. DSM Xplore $12 \mathrm{ml}$ injection molding machine was used for injection molding of compounded melt. Maximum processing and mold temperatures are 400 and $200^{\circ} \mathrm{C}$, respectively. In addition, its maximum pressure capacity is 16 bar. All the
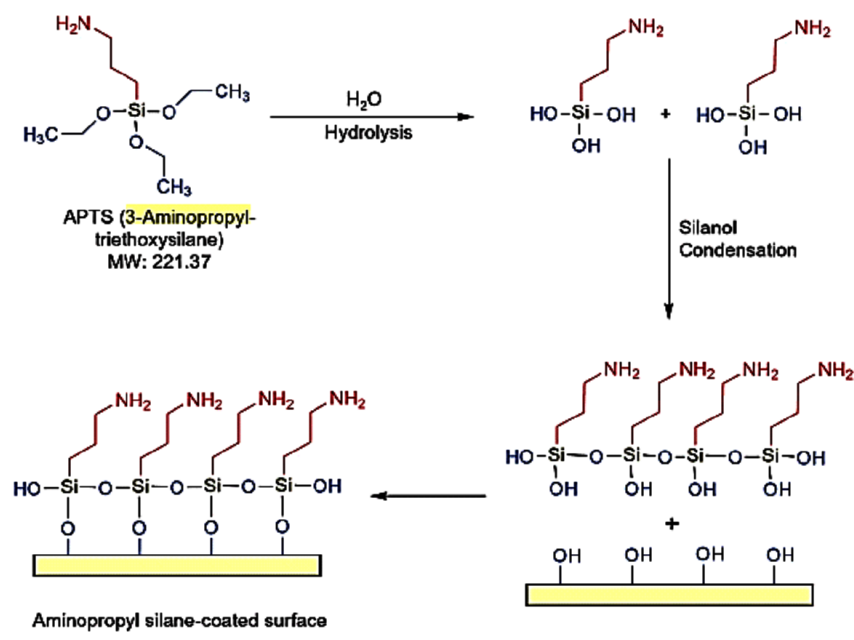

Fig. 2. Typical silane coupling flow sheet.

samples were extruded at $340^{\circ} \mathrm{C}$ of mixing temperature and $75 \mathrm{rpm}$ of screw speed and then injected into the mold at $145^{\circ} \mathrm{C}$ with an injection pressure of 12 bar.

\subsection{Dynamic mechanical analysis}

DMA tests were applied to each sample in threepoint bending mode by calibrated TA Instruments Q800 equipment at a heating rate of $5{ }^{\circ} \mathrm{C} / \mathrm{min}$ from ambient to $150^{\circ} \mathrm{C}$ under deformation frequency of $1 \mathrm{~Hz}$ and amplitude of $25 \mu \mathrm{m}$. The samples with the size of $2 \times 4 \times 27 \mathrm{~mm}^{3}$ were prepared from the molded samples. DMA measured the dynamic mechanical properties of VA/PPS composites and results were presented as storage modulus $\left(E^{\prime}\right)$ and glass transition temperature $\left(T_{\mathrm{g}}\right)$.

\subsection{SEM analysis}

SEM analysis was performed for VA/PPS samples by TESCAN VEGA 2 field emission scanning electron microscopy after gold coating.

\section{Results}

\subsection{Dynamic mechanical analysis results}

The storage modulus describes the energy stored in composite system where it is a measure of the reversible elasticity of the samples. Figure 3a illustrates the storage modulus change due to silane content.

It can be noticed that the glassy storage modulus at room temperature increases with increase of silane amount up to $3 \%$ volume content. This kind of reinforcement allowed a greater stress transfer at the interface and lead to an increase in the stiffness $[8,16] . T_{\mathrm{g}}$ was determined from the peak maximum of $\tan \delta$ and then the effect of silane content on $T_{\mathrm{g}}$ was illustrated in Fig. 3b. It is clear that silane coupling has no significant effect on $T_{\mathrm{g}}$ values of composite samples. It can be noticed that $T_{\mathrm{g}}$ of VA/PPS composites dominantly affected by matrix material of PPS instead of VA particles and the VA/PPS interface. 

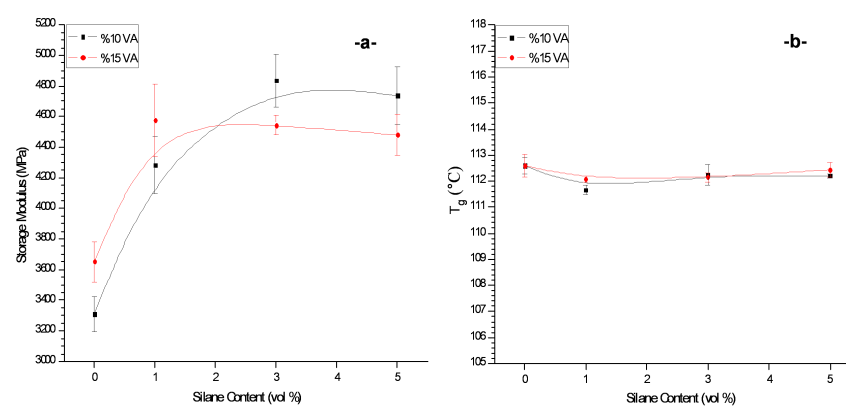

Fig. 3. Silane coupling effect of VA particles on the dynamic mechanical properties of VA/PPS composites: (a) storage modulus and (b) glass transition temperature $\left(T_{\mathrm{g}}\right)$.

\subsection{SEM results}

VA particles dispersion and adhesion with PPS of $15 \mathrm{wt} \% \mathrm{VA} / \mathrm{PPS}$ composite were investigated on bending fractured surface as shown in Fig. 4. Almost all seen particles were marked with red arrows as seen in Fig. 4a. It is clearly seen that a well distribution of VA particles in composite samples was obtained. Due to particle size, agglomeration was not expected. After silane coupling a good adhesion was obtained between VA particles and PPS matrix as clearly seen in Fig. $4 \mathrm{~b}$.

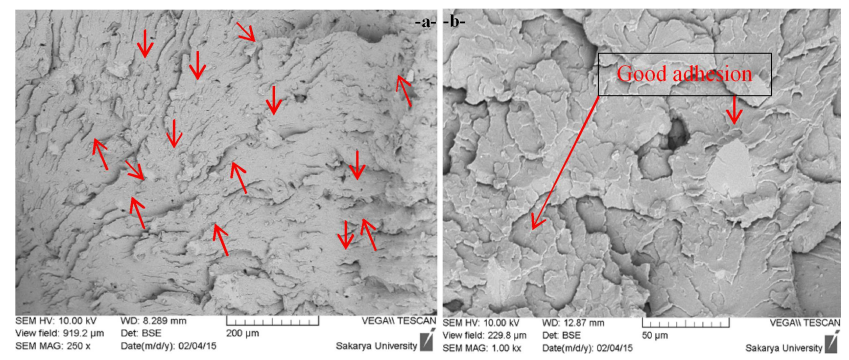

Fig. 4. Distribution and adhesion of VA particles in PPS composites: (a) original 15 wt.\% VA/PPS sample, (b) 3 vol\% silane coated 15 wt.\% VA/PPS sample.

\section{Conclusion}

Silane coupling of VA particles has been tried to be used for improving dynamic mechanical properties of VA/PPS composites and following conclusions were made:

- Storage modulus of VA/PPS composited increased with increase of silane coupling agent up to 3 vol.\% content.
- According to the SEM analysis a good relation was obtained between VA particles and PPS matrix after silane coupling.

- However, $T_{\mathrm{g}}$ values of VA/PPS composites did not change significantly by silane coupling.

\section{Acknowledgments}

This work was supported by TUBITAK, "The Scientific and Technological Research Supporting Program 1001" under contract number of 213M563.

\section{References}

[1] C.J. Schwartz, S. Bahadur, Wear 251, 1532 (2001).

[2] R.K. Goyal, K.R. Kambale, S.S. Nene, B.S. Selukar, S. Arbuj, U.P. Mulik, Mater. Chem. Phys. 128, 114 (2011).

[3] D. Wu, L. Wu, F. Gao, M. Zhang, C. Yan, Polym. Eng. Sci. 48, 966 (2008).

[4] D. Wu, L. Wu, J. Wang, Y. Sun, M. Zhang, Mater. Chem. Phys. 128, 274 (2011).

[5] A.M. Diez-Pascual, J. Guan, B. Simard, M.A. GomezFatou, Compos. Part A-Appl. Sci. 43, 997 (2012).

[6] P.P. Parlevliet, W.A.W. van der Werf, H.E.N. Bersee, A. Beukers, Compos. Sci. Technol. 68, 896 (2008).

[7] A.M. Díez-Pascual, M. Naffakh, Compos. Part A 54, 10 (2013).

[8] C.J. Schwartz, S. Bahadur, Wear 237, 261 (2000).

[9] S. Bahadur, C. Sunkara, Wear 258, 1411 (2005).

[10] E. Avcu, O. Çoban, M.Ö. Bora, S. Fidan, T. Sınmazçelik, O. Ersoy, Polym. Compos. 35, 1826 (2014).

[11] P.N. Lemougna, K.J.D. MacKenzie, U.F.C. Melo, Ceram. Int. 37, 3011 (2011).

[12] M. Shibata, R. Yosomiya, Z. Jiang, Z. Yang, G. Wang, R. Ma, Z. Wu, J. Appl. Polym. Sci. 74, 1686 (1999).

[13] J.P. Jog, N. Bulakh, V.M. Nadkarni, Bull. Mater. Sci. 17, 1079 (1994).

[14] R. Zhang, Y. Huang, M. Min, Y. Gao, X. Yu, A. Lu, Z. Lu, Polym. Compos. 30, 460 (2009).

[15] N. Ning, S. Fu, W. Zhang, F. Chen, K. Wang, H. Deng, Q. Zhang, Q. Fu, Progr. Polym. Sci. 37, $1425(2012)$.

[16] H. Skaff, T. Emrick, Angew. Chemie Int. Ed. 43, 5383 (2004).

[17] Q. Peng, D.M.Y. Lai, E.T. Kang, K.G. Neoh, Macromolecules 39, 5577 (2006).

[18] Y. Taniguchi, M. Ogawa, W. Gang, H. Saitoh, K. Fujiki, T. Yamauchi, N. Tsubokawa, Mater. Chem. Phys. 108, 397 (2008).

[19] R. Walter, K. Friedrich, V. Privalko, A. Savadori, J. Adhesion 64, 87 (1997). 